\title{
A NEW DEFINITION OF A STIELTJES INTEGRAL*
}

\author{
BY A. H. COPELAND
}

Let $f(x)$ be a function of limited variation defined on $(\alpha, \beta)$, and $x_{1}, x_{2}, \cdots, x_{n}$ a sequence which divides $(\alpha, \beta)$ into a finite number of intervals. Let $g(x)$ be bounded on $(\alpha, \beta)$. If

$$
\lim _{n \rightarrow \infty} \sum_{i=1}^{n} g\left(x_{i}\right)\left\{f\left(x_{i}\right)-f\left(x_{i-1}\right)\right\}
$$

exists when $x_{i}-x_{i-1}$ tends to zero, this limit is the RiemannStieltjes integral of $g$ with respect to $f$. If $g$ is continuous the integral exists. If $g$ is not continuous (A) need not exist. In particular (A) does not exist when $g$ is of bounded variation unless restrictions which are conditioned by $g$ are placed on the sequence $x_{1}, x_{2}, \cdots$.

In this note we define an integral of $g$ with respect to $f$ as the Cesàro mean of $g$ formed for a sequence $x_{1}, x_{2}, \cdots$ of ordinates. This sequence is defined in terms of $f$ only. When the function $f$, and consequently the method of formation of the sequence $x_{1}, x_{2}, \cdots$, has been given, the integral exists for every $g$ which has discontinuities of the first kind only, and reduces to (A) when $g$ is continuous. For the present we shall assume that $f$ is monotone, $f(\alpha+0)=0$, and $f(\beta)=1$. At a later point we shall show how these restrictions can be removed.

The sequence $x_{1}, x_{2}, \cdots$ is related to the function $f$ in the following manner: Let $a<x \leqq b$ be any sub-interval of $\alpha<x \leqq \beta$. Let $H_{n}$ be the number of points of the sequence $x_{1}, x_{2}, \cdots, x_{n}$

* Presented to the Society, December 31, 1930. For other extensions of the concept of the Stieltjes integral, see H. L. Smith, On the existence of the Stieltjes integral, Transactions of this Society, vol. 27 (1925), p. 491; S. Pollard, The Stieltjes integral and its generalizations, Quarterly Journal of Mathematics, vol. 49 (1920-3), p. 73; Kolmogoroff, Untersuchungen iiber den Integralbegriff, Mathematische Annalen, vol. 103 (1930), p. 654; Young, The algebra of manyvalued quantities, Mathematische Annalen, vol. 104 (1931), p. 260, and Many-valued Riemann-Stieltjes integration, Cambridge Philosophical Society Proceedings, vol. 27 (1931), p. 325; Ben Dushnik, On the Stieltjes integral, published by Edwards Brothers (Ann Arbor). 
which fall in $(a, b)$. Then $H_{n} / n$ is the relative frequency with which the terms of the sequence fall in $(a, b)$, and

$$
\lim _{n \rightarrow \infty} \frac{H_{n}}{n}=f(b+0)-f(a+0) .
$$

A similar condition holds for closed intervals, open intervals, and intervals closed on the left and open on the right.

Let $G_{n}=g\left(x_{1}\right)+g\left(x_{2}\right)+\cdots+g\left(x_{n}\right)$. If the sequence $x_{1}, x_{2}, \cdots$ satisfies (1) the integral is defined by*

$$
\lim _{n \rightarrow \infty} \frac{G_{n}}{n}=\int_{\alpha}^{\beta} g(x) d f(x),
$$

provided this limit exists.

In order to construct the sequence $\dagger x_{1}, x_{2}, \cdots, x_{n}$ we shall group the terms as follows: $x_{1} ; x_{2}, x_{3} ; x_{4}, x_{5}, x_{6} ; \cdots$. The $s$ th group contains the terms $x_{i}$ whose subscripts have the form $i=s(s-1) / 2+k$ where $k=1,2,3, \cdots, s$. Let $x_{i}$ be the greatest lower bound of the numbers $x$ for which

$$
f(x-0) \leqq(2 k-1) / 2 s \leqq f(x+0) .
$$

Then $f\left(x_{i}-0\right) \leqq(2 k-1) / 2 s \leqq f\left(x_{i}+0\right)$ and $a<x_{i} \leqq b$ if and only if $f(a+0)<(2 k-1) / 2 s \leqq f(b+0)$. If $z_{s}$ is the number of terms of the sth group lying in $(a, b)$, then $z_{s}$ is the number of integers $k$ for which these last inequalities hold. Simple arithmetic then gives

$[f(b+0)-f(a+0)] s-1<z_{s}<[f(b+0)-f(a+0)] s+1$.

Therefore

* $\operatorname{Lim}_{n \rightarrow \infty} G_{n} / n$ is the arithmetic or first Cesàro mean of the ordinates $g\left(x_{1}\right)$, $g\left(x_{2}\right), \cdots$. In this connection we note that J. C. Burkill has developed a definition of an integral based on Cesàro means and Perron's integral. See Proceedings of the London Mathematical Society, (2), vol. 34 (1932), pp. 314-322; vol. 39 (1935), pp. 541-552; Journal of the London Mathematical Society, vol. 10 (1935), pp. 255-259; vol. 11 (1936), pp. 43-88, 220-226.

$\dagger$ The method which we shall describe is similar to that used by von Mises in the second example of his article Über Zahlenfolgen, die ein kollektiv-ähnliches Verhalten zeigen, Mathematische Annalen, vol. 108, no. 5 (1933). An alternative construction can be found in the author's article $A$ matrix theory of measurement, Mathematische Zeitschrift, vol. 37, no. 4 (1933). 


$$
\begin{aligned}
{[f(b+0)-f(a+0)] s(s+1) / 2-s } & <h_{s} \\
& <[f(b+0)-f(a+0)] s(s+1) / 2+s,
\end{aligned}
$$

where $h_{s}=z_{1}+z_{2}+\cdots+z_{s}$. If $s(s+1) / 2 \geqq n>s(s-1) / 2$, then $h_{s-1} \leqq H_{n} \leqq h_{s}$ and hence

$$
\begin{aligned}
& {[f(b+0)-f(a+0)] s(s-1) / 2-(s-1)<H_{n} } \\
&<[f(b+0)-f(a+0)] s(s+1) / 2+s .
\end{aligned}
$$

If we divide the left-hand member of this inequality by $s(s+1) / 2$, the middle member by $n$, and the right-hand member by $s(s-1) / 2$, we obtain

$$
\begin{aligned}
{[f(b+0)-f(a+0)] } & \frac{s-1}{s+1}-\frac{2(s-1)}{s(s+1)}<\frac{H_{n}}{n} \\
& <[f(b+0)-f(a+0)] \frac{s+1}{s-1}+\frac{2}{s-1} .
\end{aligned}
$$

Therefore

$$
\lim _{n \rightarrow \infty} H_{n} / n=f(b+0)-f(a+0) .
$$

Similarly, since the number of integers $k$ satisfying the inequalities $f(a-0) \leqq(2 k-1) / 2 s \leqq f(a+0)$ is greater than $[f(a+0)$ $-f(a-0)] s-1$ and less than $[f(a+0)-f(a-0)] s+1$, it follows that the relative frequency with which a number $a$ is repeated in the sequence $x_{1}, x_{2}, \cdots$ tends to $f(a+0)-f(a-0)$ as $n$ becomes infinite. Thus conditions (1) are satisfied with respect to closed, open, and half open intervals.

We shall next prove the existence of the integral. Let $a<x \leqq b$ be any sub-interval of $(\alpha, \beta)$ and let $G_{n}{ }^{\prime}$ be the sum of those terms of the set $g\left(x_{1}\right), g\left(x_{2}\right), \cdots, g\left(x_{n}\right)$ for which the corresponding ordinates lie in $(a, b)$. Then $m H_{n} \leqq G_{n}{ }^{\prime} \leqq M H_{n}$ where $M$ and $m$ are the maximum and minimum* of $g(x)$ in $(a, b)$. Thus the superior and inferior limits of $G_{n}^{\prime} / n$ both lie between $m[f(b+0)$ $-f(a+0)]$ and $M[f(b+0)-f(a+0)]$. Hence these limits differ by at most $(M-m)[f(b+0)-f(a+0)]$. If $G_{n}^{\prime \prime}$ is the sum of those terms of $g\left(x_{1}\right), g\left(x_{2}\right), \cdots, g\left(x_{n}\right)$ for which the corresponding points of $x_{1}, x_{2}, \cdots$ are equal to some number $c$, then $G_{n}^{\prime \prime} / n$ approaches the unique limit $g(c)[f(c+0)-f(c-0)]$.

* In case $g(x)$ does not possess a maximum and minimum in $(a, b)$, then $M$ and $m$ shall designate the least upper and greatest lower bounds of $g$ in this interval. 
If $\epsilon$ is a given positive number and $c$ is any point of $(\alpha, \beta)$ for which the saltus of $g(x)$ is less than $\epsilon$, then $c$ can be enclosed in an interval $(a, b)$ within which the oscillation of $g$ is less than $\epsilon$. It follows that the superior and inferior limits of $G_{n}{ }^{\prime} / n$ differ by at most $\epsilon[f(b+0)-f(a+0)]$. If the saltus of $g$ at a point $c$ is greater than or equal to $\epsilon$, then, since the discontinuity of $g$ at $c$ is of the first kind, we can choose two points $a$ and $b$ for which the oscillation of $g$ is less than $\epsilon / 2$ in each of the intervals $a<x<c$ and $c<x \leqq b$. Hence the superior and inferior limits of $G_{n}^{\prime} / n$ for the intervals $(a, c)$ and $(c, b)$ differ respectively by at most $[f(c-0)-f(a-0)] \epsilon / 2$ and $[f(b+0)-f(c+0)] \epsilon / 2$. Since the superior and inferior limits of $G_{n}{ }^{\prime \prime} / n$ for the point $c$ differ by 0 , it follows that the superior and inferior limits of $G_{n}^{\prime} / n$ for the interval $(a, b)$ differ by at most $\epsilon[f(b+0)-f(a+0)]$.

Since $g(x)$ has discontinuities of the first kind only, it follows that the points at which the saltus of $g$ is greater than $\epsilon$ are finite in number. Consequently the interval $(\alpha, \beta)$ can be divided into a finite sum of intervals such that the superior and inferior limits of $G_{n}^{\prime} / n$ in the $i$ th interval $\left(a_{i-1}, a_{i}\right)$ of the decomposition differ by at most $\epsilon\left[f\left(a_{i}+0\right)-f\left(a_{i-1}+0\right)\right]$. It follows that the superior and inferior limits of $G_{n} / n$ differ by at most $\epsilon \sum\left[f\left(a_{i}+0\right)-f\left(a_{i-1}+0\right)\right]=\epsilon[f(\beta)-f(\alpha+0)]=\epsilon$. Thus the limit of $G_{n} / n$ exists. Moreover if $x_{1}, x_{2}, \cdots$ and $\bar{x}_{1}, \bar{x}_{2}, \cdots$ are two sequences satisfying conditions (1) with respect to $f(x)$, and if $G_{n}{ }^{\prime}$ and $\bar{G}_{n}{ }^{\prime}$ are corresponding sums for the interval $\left(a_{i-1}, a_{i}\right)$, then $G_{n}^{\prime} / n$ and $\bar{G}_{n}^{\prime} / n$ approach limits which differ by at most $\epsilon\left[f\left(a_{i}+0\right)-f\left(a_{i-1}+0\right)\right]$. It follows that $G_{n} / n$ and $\bar{G}_{n} / n$ approach the same limits.

If $f(x)$ is monotone but fails to satisfy the conditions $f(\alpha+0)$ $=0$ and $f(\beta)=1$, then we can define the integral by means of the equation

$$
\int_{\alpha}^{\beta} g(x) d f(x)=[f(\beta)-f(\alpha+0)] \int_{\alpha}^{\beta} g(x) d \phi(x),
$$

where $\phi(x)=[f(x)-f(\alpha+0)] /[f(\beta)-f(\alpha+0)]$. If $f(x)$ is an arbitrary function of limited variation, we can define the integral as the difference of the integrals with respect to the positive and negative variations of $f$. Thus if $g(x)$ has discontinuities of the first kind only and if $f(x)$ is of limited variation, then the integral exists. 
In order to form the integral over a sub-interval $\left(\alpha^{\prime}, \beta^{\prime}\right)$ of $(\alpha, \beta)$, the following device is convenient. Let $\gamma(x)$ be a function which is equal to $g(x)$ when $x$ is in $\left(\alpha^{\prime}, \beta^{\prime}\right)$ and equal to 0 otherwise. Then the integral of $\gamma(x)$ extended over the interval $(\alpha, \beta)$ is equal to the integral of $g(x)$ extended over the interval $\left(\alpha^{\prime}, \beta^{\prime}\right)$. It is however necessary to investigate whether or not this definition is consistent with (2). We have

$$
\begin{aligned}
& \int_{\alpha}^{\beta} \gamma d f=\{f(\beta)-f(\alpha+0)\} \int_{\alpha}^{\beta} \gamma d \phi, \\
& \int_{\alpha^{\prime}}^{\beta^{\prime}} g d f=\left\{f\left(\beta^{\prime}\right)-f\left(\alpha^{\prime}+0\right)\right\} \int_{\alpha^{\prime}}^{\beta^{\prime}} g d \bar{\phi},
\end{aligned}
$$

where

$$
\begin{aligned}
& \phi=\{f(x)-f(\alpha+0)\} /\{f(\beta)-f(\alpha+0)\}, \\
& \left.\bar{\phi}=\left\{f(x)-f\left(\alpha^{\prime}+0\right)\right\} /\left\{f\left(\beta^{\prime}\right)\right\}-f\left(\alpha^{\prime}+0\right)\right\} .
\end{aligned}
$$

Let $G_{n}$ and $\bar{G}_{n}$ be the sums corresponding to the first and second of these formulas respectively. Then the part of $G_{n}$ which arises from the exterior of $\alpha^{\prime}<x \leqq \beta^{\prime}$ is zero. If $(a, b)$ is an interval with $\alpha^{\prime}<a \leqq b \leqq \beta^{\prime}$, and $G_{n}^{\prime}$ and $\bar{G}_{n}^{\prime}$ are the parts of $G_{n}$ and $\bar{G}_{n}$ arising from $a<x \leqq b$, then

$$
\begin{aligned}
m\{f(b+0)-f(a+0)\} & \leqq \lim _{n \rightarrow \infty} \frac{G_{n}^{\prime}}{n} \\
& \leqq M\{f(b+0)-f(a+0)\},
\end{aligned}
$$

and the same relations hold for $\bar{G}_{n}{ }^{\prime}$. The numbers $m$ and $M$ are the greatest lower bound and least upper bound of $g$ on $a<x \leqq b$. The relation

$$
\int_{\alpha}^{\beta} \gamma d f=\int_{\alpha^{\prime}}^{\beta^{\prime}} g d f
$$

now follows as in the proof of the existence of $\int_{\alpha}^{\beta} g d f$.

If $G_{n}{ }^{1}, G_{n}{ }^{2}$, and $G_{n}$ are the sums corresponding to the functions $g_{1}(x), g_{2}(x)$, and $g(x)=g_{1}(x)+g_{2}(x)$, then $G_{n}{ }^{1}+G_{n}{ }^{2}=G_{n}$. Hence, dividing by $n$ and passing to the limit, we obtain 


$$
\int_{\alpha}^{\beta} g_{1} d f+\int_{\alpha}^{\beta} g_{2} d f=\int_{\alpha}^{\beta}\left[g_{1}+g_{2}\right] d f .
$$

In particular, if $g_{1}=g$ on $\alpha<x \leqq \gamma, g_{1}=0$ elsewhere, and $g_{2}=g$ on $\gamma<x \leqq \beta, g_{2}=0$ elsewhere, $\gamma$ being any point on $\alpha<x \leqq \beta$, it then follows from equations (4) and (5) that

$$
\int_{\alpha}^{\gamma} g d f+\int_{\gamma}^{\beta} g d f=\int_{\alpha}^{\beta} g d f,
$$

where each interval over which the integral is taken is open on the left. If $\gamma$ is a point of discontinuity of $f$ and $g(\gamma) \neq 0$, then $\int_{\gamma} g d f \neq 0$ and consequently cannot be included twice if (6) is to hold.

When $f$ is a monotone function such that $f(\alpha+0)=0$ and $f(\beta)=1$, the integral gives the average value of $g$ and hence is equal to a number $\mu$ lying between the maximum $M$ and the minimum $m$ of $g$. When the conditions $f(\alpha+0)=0$ and $f(\beta)=1$ are not satisfied, it follows from (3) that the number $\mu$ must be multiplied by $f(\beta)-f(\alpha+0)$ to give the value of the integral. Thus

$$
\int_{\alpha}^{\beta} g(x) d f(x)=\mu[f(\beta)-f(\alpha+0)] .
$$

It is easily seen that (7) is true for an arbitrary function of limited variation. By means of equations (6) and (7) it can readily be proved that the integral which we have defined reduces to the classical Stieltjes integral when $g$ is continuous.

We shall show that the formula for integration by parts,

$$
\int_{\alpha}^{\beta} d[f(x) g(x)]=\int_{\alpha}^{\beta} g(x) d f(x)+\int_{\alpha}^{\beta} f(x) d g(x),
$$

is valid provided the functions $f$ and $g$ are of limited variation and their determinations at points of singularity are such that

$$
\begin{aligned}
& f(c)=[f(c+0)+f(c-0)] / 2, \\
& g(c)=[g(c+0)+g(c-0)] / 2,
\end{aligned}
$$

for every $c$ of $(\alpha, \beta)$. First let us note that since $f$ and $g$ are of limited variation, their singularities are of the first kind only. Hence we are assured of the existence of the integrals of equa- 
tion (8). Next if $(a, b)^{*}$ is any sub-interval of $(\alpha, \beta)$, we have the equations

$$
\begin{aligned}
& \int_{a}^{b} d[f(x) g(x)]-\int_{a}^{b} g(x) d f(x)-\int_{a}^{b} f(x) d g(x) \\
& \quad=f(b) g(b)-f(a) g(a)-\nu[f(b)-f(a)]-\mu[g(b)-g(a)],
\end{aligned}
$$

where $f(a) \leqq \mu \leqq f(b)$ and $g(a) \leqq \nu \leqq g(b)$,

$$
\begin{aligned}
0=f(b) g(b)-f(a) g(a) & -\frac{f(a)+f(b)}{2}[g(b)-g(a)] \\
& -\frac{g(a)+g(b)}{2}[f(b)-f(a)] .
\end{aligned}
$$

Subtracting equation (10) from equation (9) we obtain

$$
\begin{array}{rl}
\int_{a}^{b} d[f(x) g(x)]-\int_{a}^{b} & g(x) d f(x)-\int_{a}^{b} f(x) d g(x) \\
& =\phi[g(b)-g(a)]+\gamma[f(b)-f(a)],
\end{array}
$$

where $\phi=[f(b)+f(a)] / 2-\mu$ and $\gamma=[g(b)+g(a)] / 2-\nu$. When the integration is extended over a single point $c$ (that is, from $c-0$ to $c+0)$, then the numbers $\mu$ and $\nu$ of equation (9) become respectively $f(c)$ and $g(c)$. Hence $\phi=\gamma=0$ by virtue of the determinations of $f(x)$ and $g(x)$ at their points of discontinuity. It follows that the formula for integration by parts is valid provided the interval of integration is a single point.

Returning to the general interval $(a, b)$, we observe that $|\phi|$ and $|\gamma|$ are less than or equal to the oscillations of $f(x)$ and $g(x)$ in $(a, b)$. Hence if $c$ is any point of $(\alpha, \beta)$, we can find an interval $(a, b)$ including $c$ and such that $|\phi|$ and $|\gamma|$ are less than $\epsilon / 2$ for each of the intervals $(a+0, c-0)$ and $(c+0, b+0)$. Thus

$$
\begin{aligned}
\mid \int_{a}^{b} d[f(x) g(x)]- & \int_{a}^{b} g(x) d f(x)-\int_{a}^{b} f(x) d g(x) \mid \\
& <\epsilon(|f(b)-f(a)|+|g(b)-g(a)|) .
\end{aligned}
$$

We can form a finite decomposition of $(\alpha, \beta)$ such that for each interval $(a, b)$ of this decomposition inequality (12) holds. It follows that the expression

* For the sake of simplicity $a$ is taken as a point of continuity of $f$. When this is not the case the necessary modifications are obvious. 


$$
\left|\int_{\alpha}^{\beta} d[f(x) g(x)]-\int_{\alpha}^{\beta} g(x) d f(x)-\int_{\alpha}^{\beta} f(x) d g(x)\right|
$$

is less than $\epsilon$ multiplied by the total variation of $f(x)$ plus the total variation of $g(x)$. Hence this expression must be equal to 0 and therefore the formula for integration by parts is valid under the above hypotheses.

UNIVERSITY OF MiCHIGAN

\section{A SET OF POSTULATES FOR BOOLEAN ALGEBRA}

\section{BY SOLOMON HOBERMAN AND J. C. C. MCKINSEY}

1. A New Set of Postulates. In the development of a Boolean Algebra, Boole's Law of Development

$$
f(x)=f(1) x+f(0) x^{\prime}
$$

stands out as a basic relationship. This law is so all embracing that the question naturally arises, if this is set as a postulate, what postulates in addition to it are needed to define a Boolean Algebra? Using as undefined a class $K$ and the Sheffer stroke function, we shall show that, in addition to a form of Boole's Law, only two "trivial" postulates are required.

\section{Postulates.*}

I. $K$ contains at least two elements.

II. If $a$ and $b$ are elements of $K$, then $a / b$ is an element of $K$.

Definitions: $a^{\prime}=a / a, a \cdot b=a^{\prime} / b^{\prime}$, and $a+b=(a / b)^{\prime}$.

III. There exists in $K$ a unique element 0 , such that, if $f(x)$ is any function definable in terms of/and elements of $K$, we have, for any $x$ in $K$,

$$
f(x)=f\left(0^{\prime}\right) x+f(0) x^{\prime} .
$$

TheOREM 1. $0^{\prime \prime}=0$.

Proof: From III, and the preceding definitions, we have

$$
x=0^{\prime} x+0 x^{\prime}=\left[\left(0^{\prime} x\right) /\left(0 x^{\prime}\right)\right]^{\prime} ;
$$

in particular

* This is the smallest set of postulates for a Boolean Algebra yet given. 